











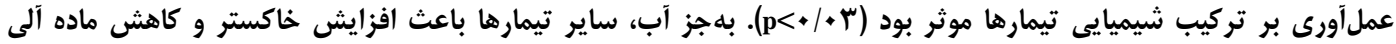
















فرنكَى در شرايط آزمايشكاهى موثر بودند.

وازههاى كليدى: آسيرزيلوس نايجر، ارزش تغذيهاى، بقاياى نخود زراعى، تريكودرما هارزيانوم، براكسيد هيدروزن، هيدروكسيد سديم، قارج صدفى نايجى ارثن

عملآورى سبب افزايش خاكستر، كاهش ماده آلى، افزايش




































مقدار يروتئين خام، و كاهش الياف خام، ديواره سلولى فاقد إندا







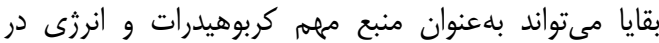













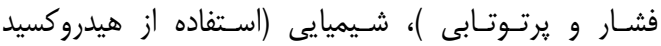
















هيدروزن بر ارزش تغذيهاى بقاياى ماش مشاهده كردنان 


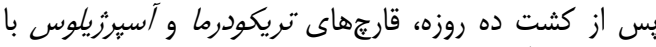

















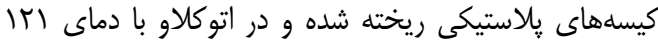







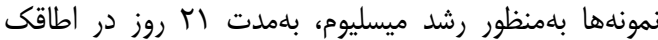

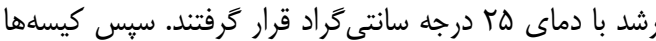

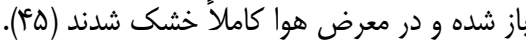
تعيين تركيب شيميايى

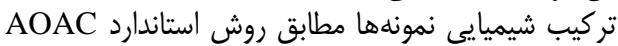











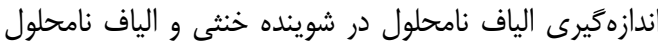







${ }^{\Delta} \mathrm{TDN}=\Lambda \mathrm{N} / \mathrm{\mu}+\left(\mathrm{CP} \times \cdot /{ }^{\sim} \mathcal{}\right)-(\mathrm{ADF} \times \cdot / \mathrm{V})$

${ }^{\wedge} \mathrm{NEL}=(\cdot / \cdot \mathrm{TF} \Delta \times \mathrm{TDN})-\cdot / \mathrm{T}$

${ }^{\gamma} \mathrm{NEg}=(\cdot / \cdot r q \times \mathrm{TDN})-\mathrm{y} \cdot 1$

آز آزمون توليد كَاز

توليد كاز تيمارهاى آزمايشسى بـر اسـاس روش اسـتاندارد



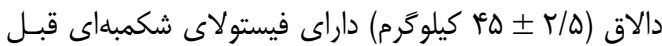























همىسلولز، همى سلولز و ليخنين در نتيجه استفاده از

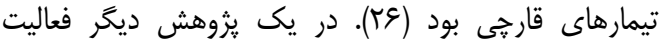



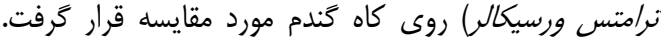






متابوليسم افزايش يافت (بآماء).



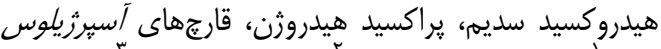


شيمايى، فراسنجههاى توليد كاز بقاياى نخود زراعى بود.

 تهيه و عمل آورى بقاياى نخود زياء زراعى}



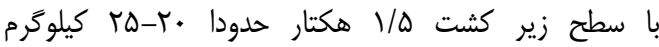













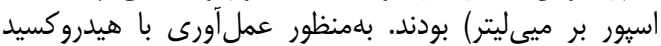

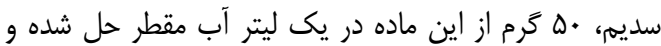













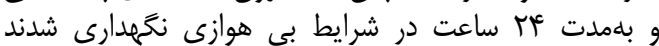





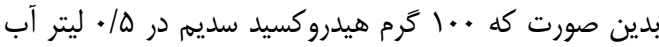

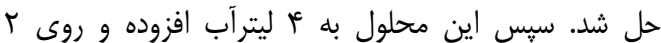

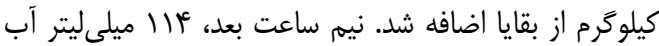





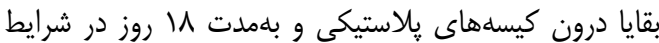

















\begin{tabular}{|c|c|c|}
\hline 1- Aspergillus Niger & 2- Trichoderma harzianum & 3- Pleurotus ostreatus \\
\hline
\end{tabular}
6- Natural Energy lactation (NEL) 7- Natural Energy growth (NE 
از فاز مايع جدا شدند. سيس pH فاز مايع نمونهها اندازهيـرى





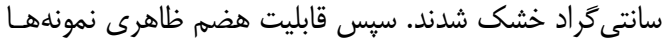

$$
\text { محاسبه شد. }
$$

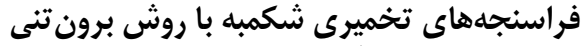











(رابطه (ر) (ر) (ر)

MB (ميلى گرم) = GP × (PF - T/T)

در اين رابطه، MB توليد توده ميكروبى،


(ميلى

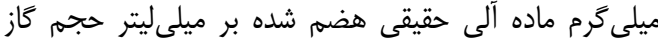






تجزيه آمارى داده دانها

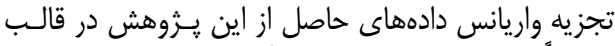



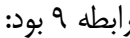
$\mathrm{Y}_{\mathrm{ij}}=\mu+\mathrm{T}_{\mathrm{i}}+\mathrm{e}_{\mathrm{ij}}$










حداقل تفاوت معنىدار ' استفاده شد.

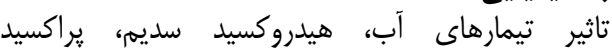









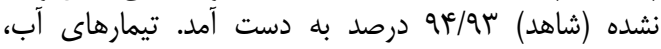



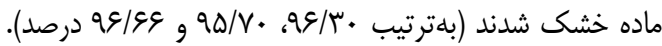



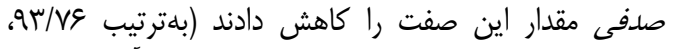



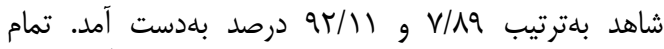
تيمارها باعث افزايش مقدار خاكستر و كاهش مادئ واده آلى شدند.

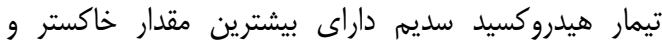

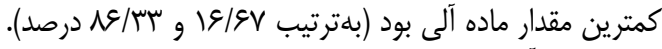









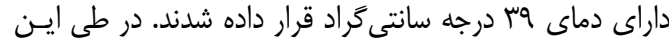



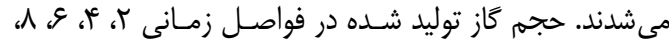







$\mathrm{y}=\mathrm{b}\left(1-\mathrm{e}^{-\mathrm{ct}}\right)$

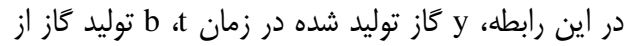

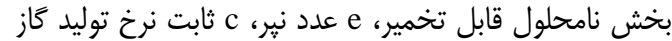
براى بخش b و أ زمان كشت هستند.













$\mathrm{ME}=r / 4++\cdot / \mu \mathrm{GP}+\cdot / \cdot \Delta V \mathrm{CP}+\cdot / \cdot+r q \mathrm{CF}$


(رابطه (ر) (ر)

SCFA $=\cdot 1 \cdot$ rTHGP - + + rTA (V (رابطه)

در اين روابط: ME: انرزى قابل متابوليسم (معازول در

























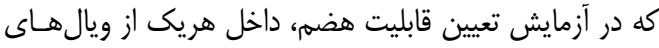









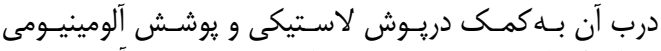





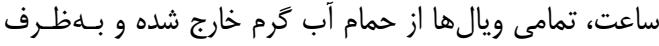

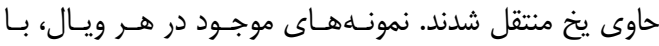




عمل آورى با آهك افزايش يافت، شايد بتوان تاثير قابل





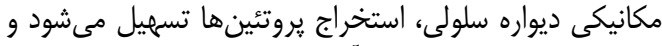





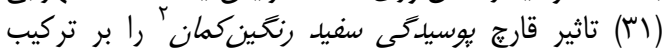



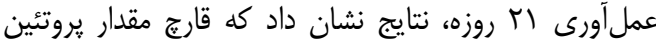

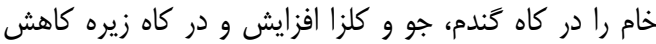

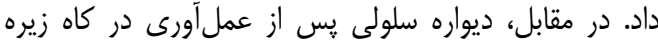

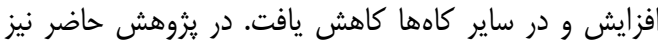

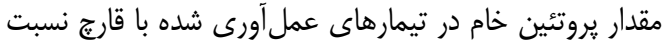

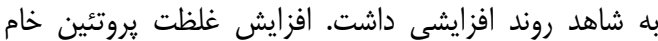

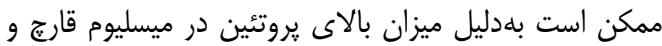

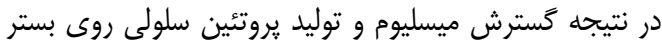

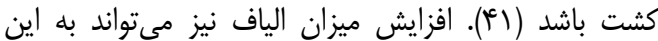





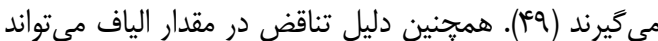

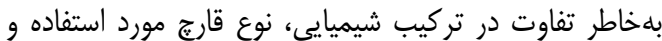



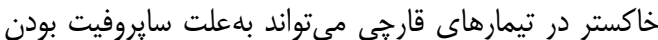





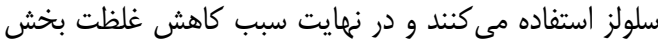

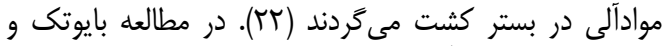



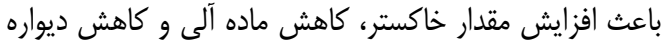






باشد. كاهش در مقدار ماده آلى نيز نتيجه رقيميل رقيق شدن مواد قندى درنتيجه افزايش مقدار خاكستر مى باشد.









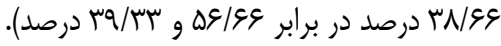







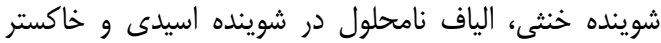

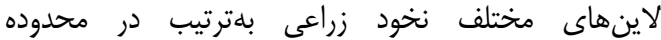

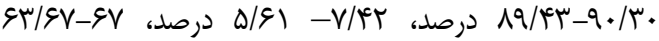

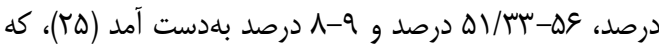

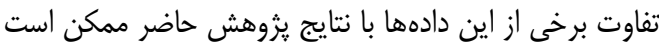

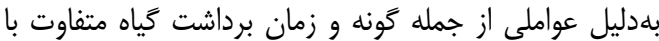

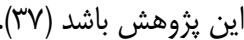
از آنجايى كه در عمل آورى با براكسيد هيدريد هيدروثن، ابتدا

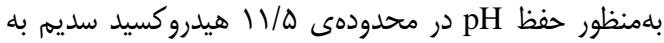



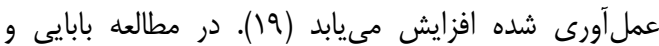


سبب افزايش خاكستر شد كه با نتايج يزوهش حاض حاض مراكر مطابقت

كاهش مقدار ماده خشك در طى فر آيند تخمير با قارجها

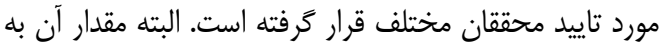



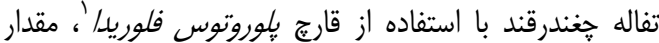

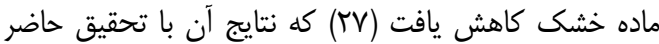











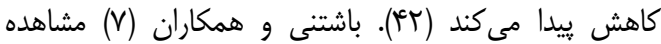

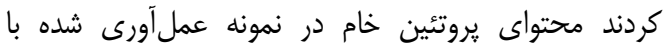
هيدروكسيد سديم در مقايسه با نمونه عمل آورى نشده ورى 
جدول ا- تاثير عمل آورى شيميايى و بيولوزيكى برتر كيب شيميايى بقاياى نخود زراعى Table 1. Effect of chemical and biological processing on chemical composition of cicer arietinum waste

\begin{tabular}{|c|c|c|c|c|c|c|c|c|c|}
\hline $\mathrm{NEg}$ & $\mathrm{NE}_{\mathrm{L}}$ & TDN & ADF & NDF & $\mathrm{CP}$ & $\mathrm{OM}$ & Ash & DM & تيمارها \\
\hline$\cdot / \omega^{a b}$ & $1 / 18^{\mathrm{ab}}$ & $\Delta T / T^{a b}$ & $r q / \mu^{\prime a b c}$ & $\Delta \xi / q^{a b c}$ & $r / r q^{e}$ & $9 r / 11^{a}$ & $V / 19^{c}$ & $q \uparrow / q \mu^{c}$ & شاهد \\
\hline.$/ 4 \Lambda^{\mathrm{b}}$ & $1 / 1 F^{b}$ & $\Delta) / \Delta \cdot{ }^{b}$ & $r \cdot / 99^{\mathrm{ab}}$ & $\Delta N / .^{a b c}$ & $r / 99^{d}$ & १) $/ \Delta \Delta^{\mathrm{a}}$ & $N / F \kappa^{c}$ & $a s / \mu \cdot{ }^{a}$ & آب \\
\hline.$/ \leftarrow^{\mathrm{b}}$ & $1 / / r^{b}$ & $0 . / 99^{b}$ & $F r / . .^{a}$ & $\Delta f / . .^{d}$ & $F / \Delta \Lambda^{b}$ & c c/ c & $\mid \varepsilon / q \mathrm{~V}^{\mathrm{a}}$ & $q \Delta / v \cdot{ }^{b}$ & هيدرو كسيد سديم \\
\hline$\cdot / \Delta r^{\mathrm{ab}}$ & $1 / 1 v^{a b}$ & $\Delta r / \Lambda \omega^{a b}$ & $\Gamma / g q^{b c}$ & $\Delta s / .^{c d}$ & $r / \& q^{\mathrm{e}}$ & $\Lambda \varepsilon / 4 \varphi^{c}$ & $\mid r / \Delta \omega^{\mathrm{a}}$ & $৭ \& / 99^{\mathrm{a}}$ & يراكسيد هيدروثن \\
\hline$\cdot / \leftarrow \Lambda^{\mathrm{b}}$ & $1 / / F^{b}$ & $\Delta I / V^{b}$ & $r \cdot / 9 g^{\mathrm{ab}}$ & $\Delta s / . .^{c d}$ & $f / v \varphi^{\mathrm{a}}$ & $q . / . .^{b}$ & $1.1 . .^{b}$ & $q \Psi / v \varsigma^{d}$ & قارج تريكودرما \\
\hline$\cdot / \Delta \mathrm{r}^{\mathrm{a}}$ & $|/ r|^{\mathrm{a}}$ & $\Delta F / \xi \Delta^{a}$ & 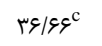 & $\Delta q / \Psi^{\mathrm{ab}}$ & $F / \Delta \Lambda^{b}$ & $q \cdot / 11^{b}$ & $9 / 19^{b}$ & $q \Psi / \Upsilon^{\Psi^{d}}$ & قارج آسيرزيلوس \\
\hline.$/ F \Delta^{b}$ & $1 / 1^{b}$ & $\Delta \cdot / \Delta \Delta^{b}$ & $\kappa r / . .^{a}$ & $9 \cdot 1 . .^{\mathrm{a}}$ & $r / T \cdot{ }^{c}$ & $\wedge a / \gamma^{b}$ & $1 . / r^{b}$ & $q \Psi / N \mathcal{V}^{d}$ & قارج صدفى \\
\hline . $1.4 t$ &.$/ .4$. & - / $99 \Delta$ & ג.r. & ג &.$/ \Delta T$ & . / rVa & $\cdot /$ TVA & . & اشيباه معيار \\
\hline.$/ . r \Delta F$ & . /. rQf &.$/ . T^{\top} \Delta F$ & . $1 . r \& 9$ & . & $<\cdot / \ldots+\mid$ & $<\cdot \mid \cdots+$ & $<\cdot|+\cdots|$ & $<\cdot|+. \cdot|$ & احتمال معنى دارى \\
\hline
\end{tabular}

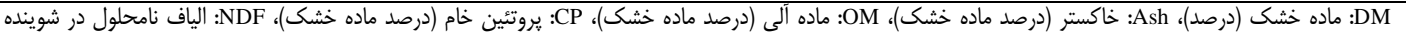

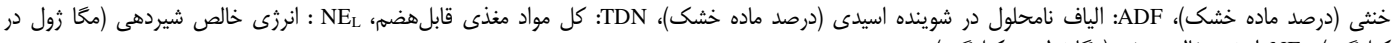

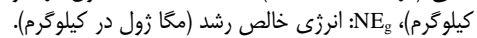

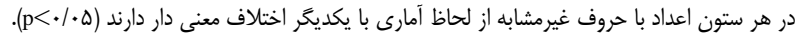

تيمارهاى هيدروكسيد سديم و قارج صدفى و همجنين

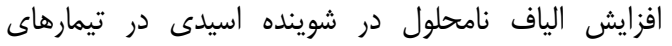

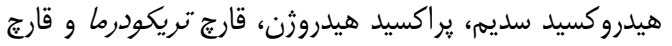

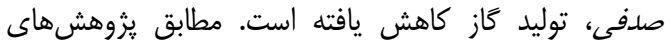

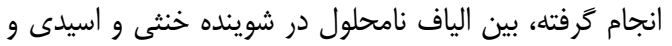

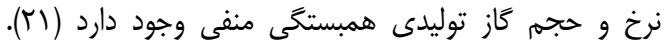

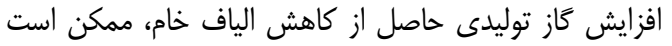

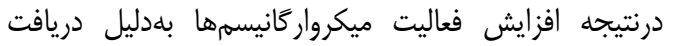

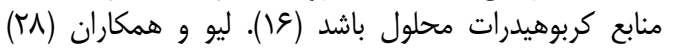

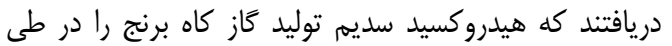

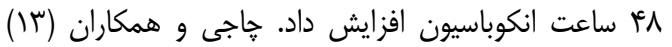

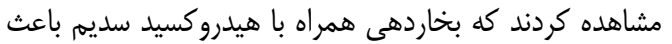

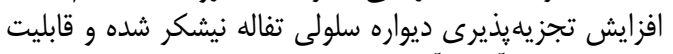

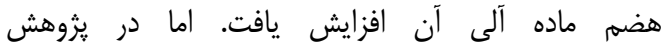
دانشمسگران و همكاران (ع) (1)، قابليت هضم ماده آلى كانى كاه

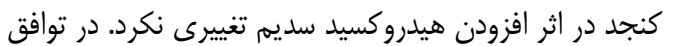

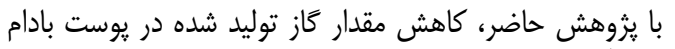

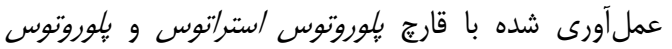

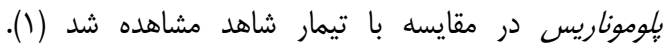

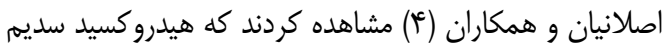

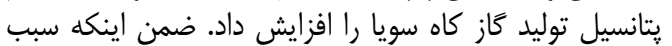

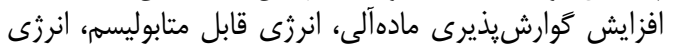

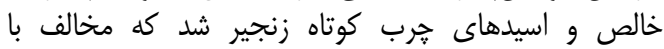

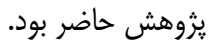

قابليت هضم و فراسنجه بوداى تخميرى آزمايشخَاهى

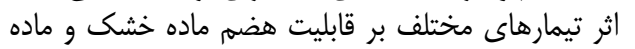

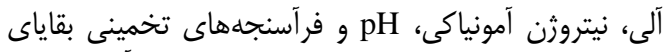

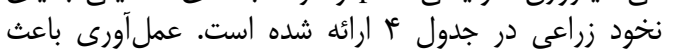

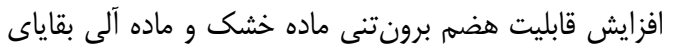

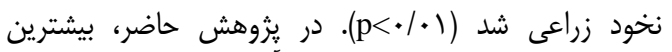

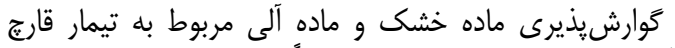

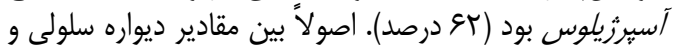

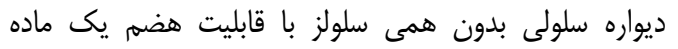

توليد كاز و كينتيك توليد كَاز

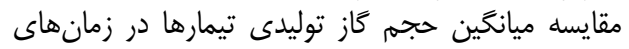

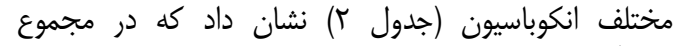

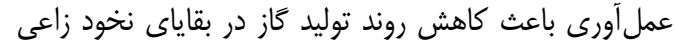

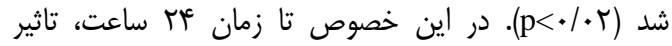

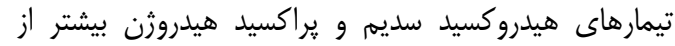

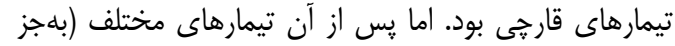

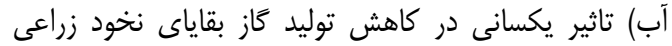
داشتند.

فراسنجههاى توليد كاز و تخمينى بقاياى نخود زراعى إنى

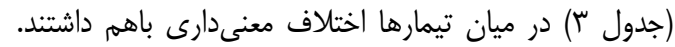

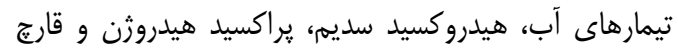

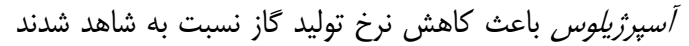

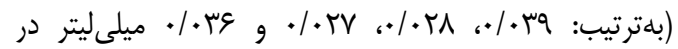

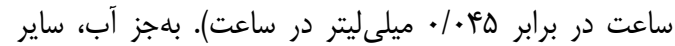

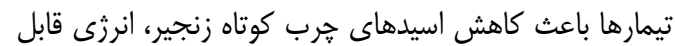

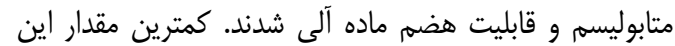

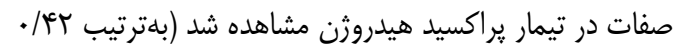

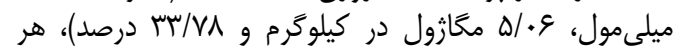

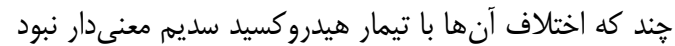

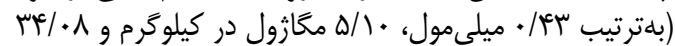

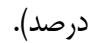

اندازهزيرى كاز توليدى در شرايط برونتنى اطلاعات

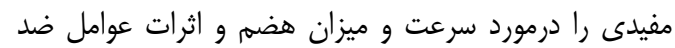

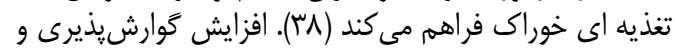

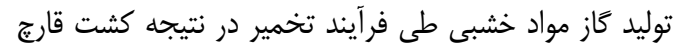

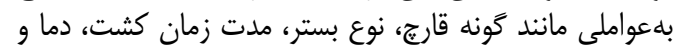

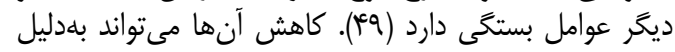

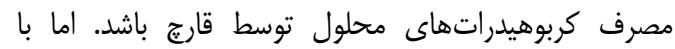

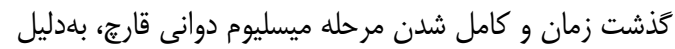

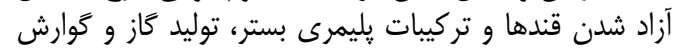

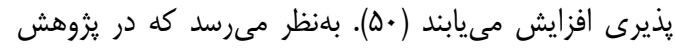
حاضر نيز با افزايش ميزان الياف نامحلول در شوينده خنتى در 


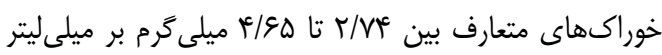

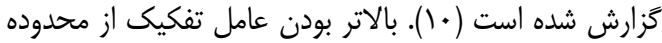

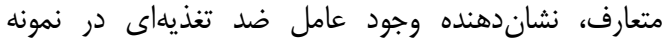

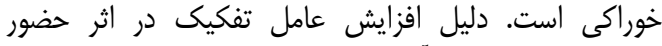

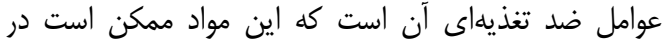

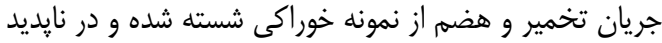

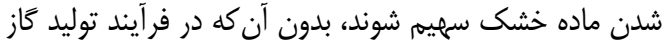

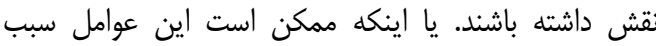

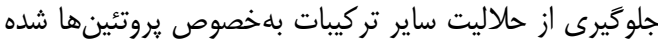

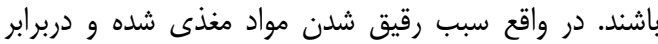

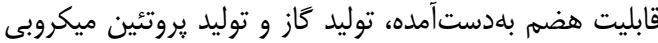

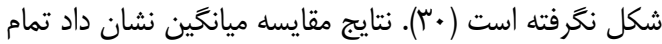

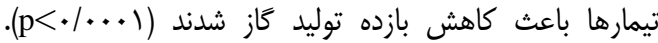

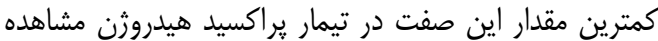

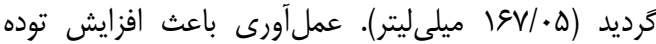

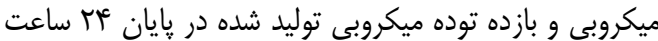

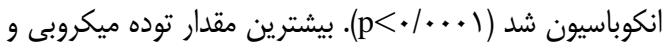

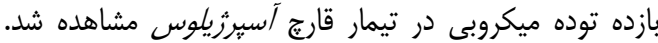

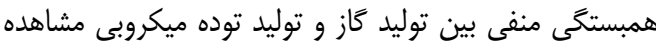

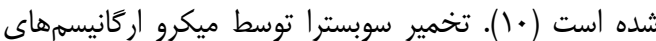

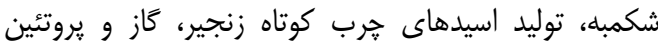

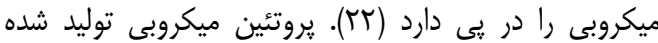

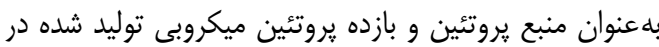

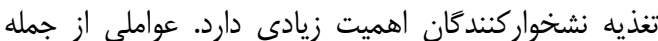

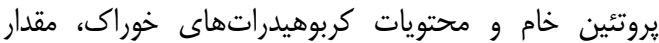

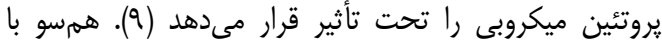

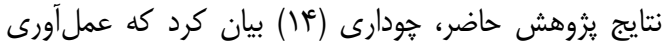

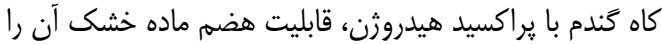

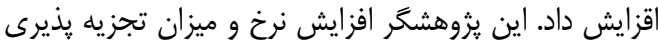

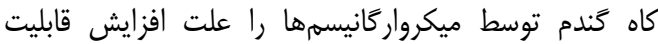

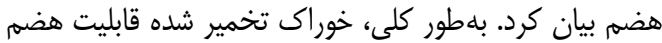

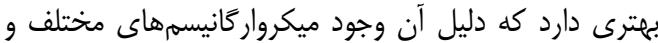

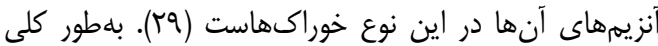

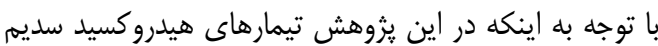

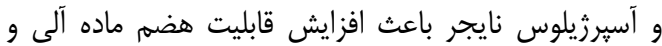

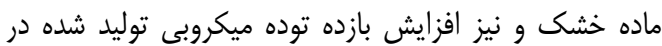

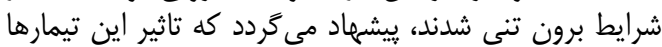

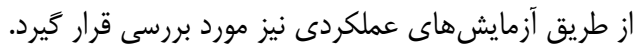

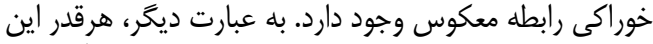

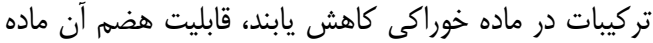

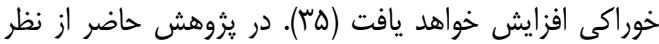

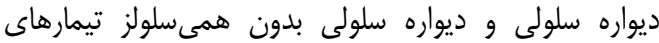

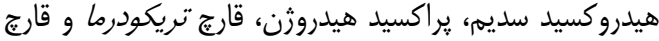

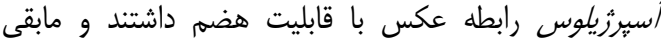

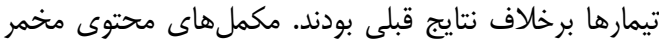

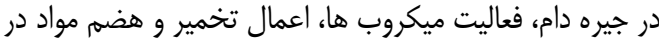

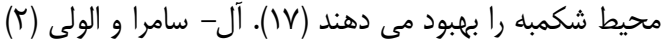

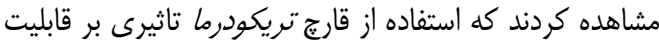

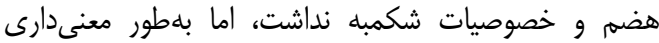

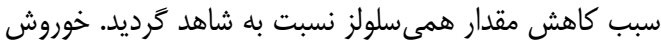

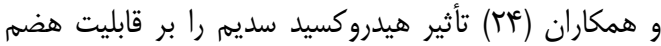

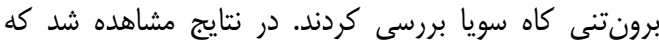

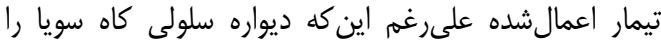

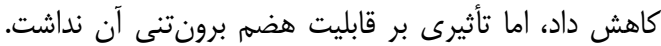

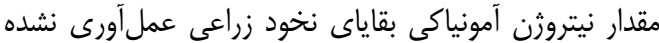

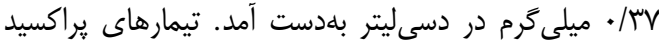

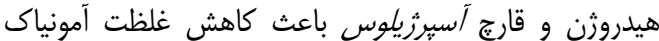

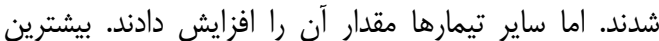

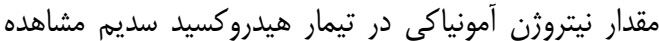

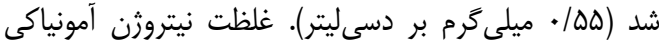

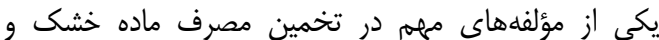

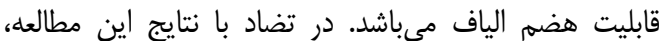

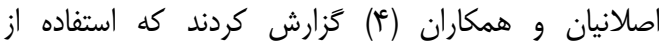

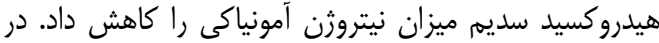

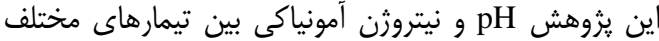

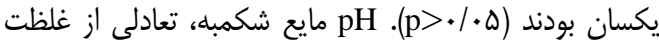

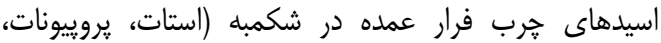

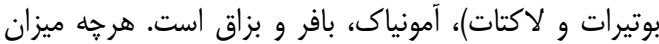

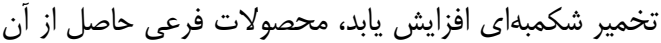

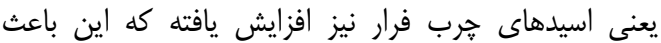

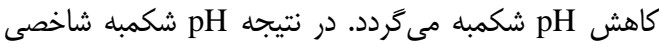

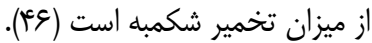

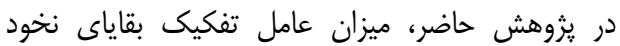

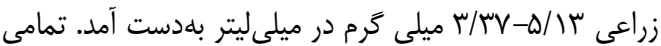

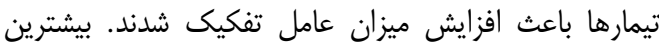

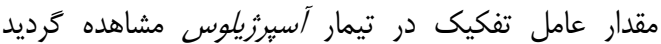

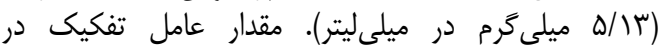


جدول r- تاثير عمل آورى شيميايى و بيولوزيكى بر روند توليد كاز بقاياى نخود زراعى در زمانهاى مختلف انكوباسيون (ميلىليتر در كرم ماده خشك) Table 2. Effect of chemical and biological processing on in vitro gas production of cicer arietinum wastes at different times of incubation (ml/g DM)

\begin{tabular}{|c|c|c|c|c|c|c|c|c|c|c|}
\hline 99 & vr & $i \wedge$ & ع & MF & IT & $\wedge$ & 8 & r & $r$ & تيمار \\
\hline$r \cdot r / \mu \mu^{a}$ & $M N / T r^{a}$ & $198 / 9 \mathrm{~V}^{\mathrm{a}}$ & $|Q| / \& V^{a}$ & $\mid r N / \mu^{a}$ & $91 / 9 \mathrm{~V}^{\mathrm{a}}$ & $V \Delta / .^{a}$ & $\Delta \mathcal{N} / \mu^{\mathrm{a}}$ & $r \varepsilon / q v^{\mathrm{a}}$ & $\mid N / r^{a}$ & شاهد \\
\hline $19 \Lambda / \Gamma^{2}$ & $\mid \Lambda r / r^{a b}$ & $|q| / 9 \mathrm{v}^{\mathrm{ab}}$ & $\mid f \& / \& \mathrm{~V}^{\mathrm{ab}}$ & $\mid r g / g V^{a}$ & $\Lambda \cdot / .{ }^{\mathrm{b}}$ & b b/r/ b & (c) & $r \varepsilon / \& \mathrm{~V}^{\mathrm{b}}$ & $q / q V^{b c}$ & آب آب \\
\hline $\mid V r / r r^{c}$ & $\mid Q S / \& V^{c}$ & $1 f \cdot / .^{c}$ & Irr/rde & $q \& / q V^{\mathrm{cd}}$ & 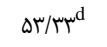 & $|i| / \& V^{c}$ & $r \Delta / . .^{d}$ & |r/r/r & $1 / q V^{c}$ & هيدروكسيد سديم \\
\hline $\mid S N / \Gamma^{c}$ & $10 \cdot / .^{c}$ & Irr/r/re & $111 / q V^{e}$ & 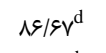 & $\kappa r / r r^{d}$ & $r V / q V^{c}$ & $M V / q V^{d}$ & $1.1 . .^{c}$ & $\% .^{c}$ & يراكسيدهيدروزن \\
\hline $\mid V \varepsilon / q V^{c}$ & $\mid \omega \& / \& V^{c}$ & $\mid \kappa r / \Psi^{c}$ & $\mid r \varepsilon / q \mathrm{~V}^{\mathrm{cd}}$ & $1 \cdot N / r^{b c}$ & $\mathrm{VN} / \mathrm{rr}^{\mathrm{bc}}$ & $99 / 9 \mathrm{v}^{\mathrm{ab}}$ & $\Delta \Gamma / \Psi^{\mathrm{ab}}$ & $r \varepsilon / q v^{a}$ & $\mid q / 9 v^{\mathrm{a}}$ & قارج تريكودرما \\
\hline $\mid V \Delta / .^{c}$ & $\mid Q N / r r^{c}$ & $|f| / g V^{c}$ & $|r| / \& V^{\mathrm{de}}$ & $1.1 / 9 \mathrm{v}^{\mathrm{bc}}$ & $9 N / \mu^{c}$ & $\Delta \mathrm{N} / \Gamma^{\mathrm{b}}$ & $\varphi_{\phi} \Delta / . .{ }^{\mathrm{bc}}$ & $r \mid / q v^{a b}$ & (r/ & قارج آسبرزيلوس \\
\hline $14 \cdot / . .^{b c}$ & $\mid 9 \Delta / . .{ }^{b c}$ & $\mid f N / r^{b c}$ & $\mid r \Delta / . . b c$ & $11 \% / \Gamma^{b}$ & 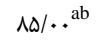 & $99 / 9 \mathrm{v}^{\mathrm{ab}}$ & $\Delta \Gamma / \Psi^{\mathrm{ab}}$ & $r N / r \mu^{a}$ & $\mid N / r^{a}$ & قارج صدفى \\
\hline s/৭ఎV & V/ITS & D/DST & F/mGG & r/Q人ץ & r/VYG & $r / 91 \Lambda$ & $r / r M I$ & T/MAS & r/Frq & اشتباه معيار ميانغين \\
\hline . &.$/ .14 F$ & $\% 1+v_{9}$ &.$|\cdots|$ & $<\cdot / \ldots \mid$ & $<\cdot|\cdots|$ & $<\cdot|\cdots \cdot|$ & $<\cdot / \ldots)$ & $<\cdot|\cdots|$ & . $/$ r & اختلاف معنىدارى \\
\hline
\end{tabular}

Table 3. Effect of chemical and biological processing on gas production parameters of cicer arietinum wastes

\begin{tabular}{|c|c|c|c|c|c|}
\hline قابليت هضم ماده آلى (درصد) & 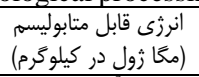 & 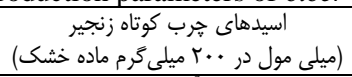 & 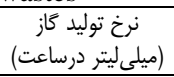 & يتانسيل توليد كاز & 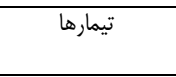 \\
\hline$r V / q V^{a}$ & $\Delta / 8 q^{a}$ & $\cdot / \Delta \AA^{\mathrm{a}}$ & $\cdot / \cdot \theta \cdot \pm \cdot / \cdot+r$ & $19 r \pm 4 / \% r$ & 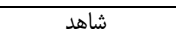 \\
\hline$r V / g V^{a}$ & $\Delta / \& \varphi^{\mathrm{a}}$ & $\cdot 1 \Delta \Delta^{\mathrm{a}}$ & $. / . r \mid \pm . / \cdot r t$ & $19 \Delta / \Lambda \pm \Gamma / \Lambda \Delta$ & 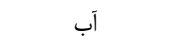 \\
\hline$r T / q^{c a}$ & $r / \Lambda r^{c a}$ &.$/ e^{\mathrm{ca}}$ & $. / . r q \pm . / . r r$ & $\mid \Lambda T / T \pm \Delta / 4 T$ & هيدروكسيدسديم \\
\hline$r \cdot / \& \Lambda^{\mathrm{u}}$ & $\varphi / \Delta \Delta^{\mathrm{u}}$ & $\cdot / \sim^{u}{ }^{u}$ & $. / \cdot r t \pm . / . r t$ & س & يراكسيدهيدروثن \\
\hline$m e / r^{b c}$ & $\Delta / / f^{b c}$ &.$/ \mathrm{er}^{\mathrm{bc}}$ & $. / \cdot \Delta r \pm . / . \cdot F$ & $195 / q \pm F / q 4$ & قارج تريكودرما \\
\hline$r / M \Lambda^{b c}$ & $F / q \varepsilon^{b c}$ & $\cdot / \mathrm{cF}^{\mathrm{bc}}$ & $.|\cdot t| \pm . / \cdot r$ & $199 \pm \Delta / r r$ & قارج آسيرزيلوس \\
\hline$r \Delta / r \Lambda^{b}$ & $\Delta / Y \Lambda^{b}$ &.$/ 4 q^{b}$ & 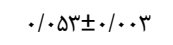 & $\mid \& N / \Delta \pm \Psi / \varnothing 9$ & قارج صدفى \\
\hline$\cdot / V / D$ & $\cdot 11 \cdot V$ &.$/ .1 \mathrm{~V}$ & - & - & اشتباه معيار ميانكين \\
\hline$<. / \cdots+$ & $<\cdot / \cdots+$ & $<\cdot / \cdots+$ & - & - & اختلاف معنىدارى \\
\hline
\end{tabular}


جدول ع- تأثير عمل آورى شيميايى و بيولوزيكى بر قابليت هضم ماده خشك و ماده آلى، نيتروزن آمونياكى، pH و فراسنجههاى تخميرى بقاياى نخود زراعى Table 4. Effect of chemical and biological processing on dry matter and organic matter digestibility, ammoniacal nitrogen, pH and estimated parameters of cicer arietinum wastes

\begin{tabular}{|c|c|c|c|c|c|c|c|c|}
\hline 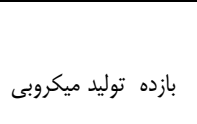 & (ميلى تور بر كرمر ماده خشكى) & (ميلى ليتر بر كرم ماده خشكى) & (ميلى كرم برميلى ليتر) & $\mathrm{pH}$ & (ميلى كرم بر دسى ليتر) & قابليت هضم ماده آلى & ماده خشكى & تيمارها \\
\hline.$/ \mu \mu^{c}$ & $\Lambda \mu^{\prime} / q^{\mathrm{d}}$ & $r 91 / \cdot v^{a}$ & $r / r v^{c}$ & $\varepsilon / 4 \Delta^{\mathrm{a}}$ &.$/ T r$ & $\Delta r^{c}$ & $\Delta F^{c}$ & شاهد ل كاهد \\
\hline $.1 \% b^{b}$ & $11 . / 9 \mathrm{C}^{\mathrm{c}}$ & $r \mid s / g V^{b}$ & $\% / . r^{b}$ & $s / r^{b}$ &.$/ 0 \mathrm{r}$ & $\Delta r^{b c}$ & $\Delta \delta^{b c}$ & آب \\
\hline$\cdot / \Delta \Delta^{a}$ & IrN/rrab & $1 \mathrm{~V} \cdot / \mathrm{eq}^{\mathrm{c}}$ & $r / q v^{a}$ & $s / \kappa r^{\mathrm{ab}}$ &.$/ \Delta \infty$ & $\Delta \mathrm{v}^{\mathrm{ab}}$ & $\Delta \Lambda^{b}$ & هيدروكسيدسديم \\
\hline.$/ \Delta f^{a}$ & $|r| / \cdot \lambda^{\mathrm{bc}}$ & $\mid 9 V 1 / 0^{\circ}$ & $F / M^{a}$ & $s / \kappa r^{\mathrm{ab}}$ & . & $\Delta \mathrm{b}^{\mathrm{bc}}$ & $\Delta \Lambda^{b}$ & ت براكسيدهيدروزن \\
\hline.$/ 4 \Lambda^{b}$ & $1 \pi r / \lambda \cdot \mathrm{bc}$ & $r \cdot V / \Delta r^{b}$ & $r / r r^{b}$ & $\varepsilon / \kappa^{\mathrm{ab}}$ &.$/ 44$ & $\Delta \mathrm{s}^{\mathrm{bc}}$ & $\Delta \mathrm{r}^{\mathrm{bc}}$ & قارج تريكودرما \\
\hline$\cdot / \Delta \mathrm{r}^{\mathrm{a}}$ & $19 . / r \Lambda^{\mathrm{a}}$ & $|V E / \Delta|^{c}$ & $\Delta / 1 r^{\mathrm{a}}$ & $s / r^{b}$ &.$\mu F$ & $q \tau^{\mathrm{a}}$ & $q r^{\mathrm{a}}$ & قارج آسيرزيلوس \\
\hline.$/ \mu \Lambda^{b}$ & $\| F / \pi r^{c}$ & $r \cdot F / \Lambda)^{b}$ & $k / \pi r^{b}$ & $s / r^{b} \Lambda^{b}$ & . & $\Delta r^{b c}$ & $\Delta \Delta^{b c}$ & قارج صدفى \\
\hline .1 .19 & $V / V \backslash \Lambda$ & $D / A \cdot T$ & . MST & .1 .19 & .1 .94 & .1 .19 &.$/ .14$ & اشتباه معيار ميانكيين \\
\hline$\langle\cdot|\ldots|$ & $\%$ & $<\cdot / \cdots)$ & $<\cdot|\cdots|$ & . NFAT & $\cdot / \Delta \Delta 19$ & $\% . .91$ & .1 .1115 & 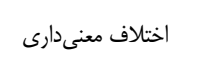 \\
\hline & & & & & \multicolumn{4}{|c|}{ "تر هر ستون، اعداد با حروف غيرمشابه از لحاظ آمارى با يكديكر اختلاف معنىار دارند (ه./.<p). } \\
\hline
\end{tabular}



white-rot fungi: Pleuro-tus ostreatus and Pleurotus pulmonarius. African Journal of Biotechnology, 9(11): 1706-1712.

2. Al-Samaraae, W.H. and S.N. Alwaeli. 2016. Effect of treated barley straw with Trichoderma harzianum fungi in some productive characteristic, 5(4): 44-48.

3. AOAC. 2005. Official methods of analysis. Association of official analytical chemists. Washington, DC. USA.

4. Aslanian, A., F. Ghanbari, J. Bayat Kouhsar and B. Karimi Shahraki. 2015. Effects of processing with gamma ray, sodium hydroxide and calcium oxide on gas production parameters and digestibility of soybean straw. Journal of Animal Productions 2: 235-248 (In Persian).

5. Azizi-Shotorkhoft, T., H. Mohammadabadi, M. Motamedi Chaji and H. Fazaeli. 2016. Isolation and identification of termite gut symbiotic bacteria with lignocellulose-degrading potential and their effects on the nutritive value for ruminants of some by-products. Animal Feed Science and Technology, 1-31.

6. Babayi, M., F. Ghanbari, A.M. Gharehbash and J. Bayat Kouhsar. 2016. Effects of processing with electron beam, hydrogen peroxide and hydrobromic acid on the nutritional value of vetch wastes. Iranian Journal of Animal Science Research, 8(3): 441-454 (In Persian).

7. Bashtani, M., J. Farzadmehr, O. Ghafori, N. Afzali and M. Sharifi. 2015. Effect of growth stage and processing by $\mathrm{NaOH}$ and $\mathrm{CaOH} 2$ on chemical composition and degradation parameters of Haloxylon $s p$. pasture plant in seeding stage. Research on Animal Production, 6(12): 96-104 (In Persian).

8. Baytok, E., T. Aksu, M.A. Karsli and H. Muruz. 2005. The effects of formic acid, molasses and inoculant as silage additives on corn silage composition and ruminal fermentation characteristics in sheep. Turk J Vet Animal Science, 29: 469-474.

9. Blummel, M. and E.R. Orskov. 1993. Composition of in vitro gas production and nylon bag degradability of roughages in predicting food intake in cattle. Animal Feed Science and Technology, 40: 109-119.

10. Blummel, M., P.S. Makkar and K. Becker. 1997. In vitro gas production: a technique revisited. Journal of Animal Physiology and Animal Nutrition, 77: 24-34.

11. Bouchard, J., M. Methot and B. Jordan. 2006. The effects of ionizing radiation on the cellulose of woodfree paper. Cellulose, 13: 601-610.

12. Broderick, G.A. and J.H. Kang. 1980. Automated simultaneous determination of ammonia and total amino acids in ruminal fluid and in vitro media. Journal of Animal Science, 63: 64-75.

13. Chaji, M., T. Mohammadabadi, M. Mamouie and S. Tabatabaei. 2010. The effect of processing with high steam and sodium hydroxide on nutritive value of sugarcane pith by in vitro gas production. Journal of Animal and Veterinary Advance, 9: 1015-1018.

14. Chaudhry, A.S. 1997. Washing and filtration of wheat straw treated with sodium hydroxide alone or with hydrogen peroxide to modify cell wall composition and in vitro digestibility. Australasian Journal of Agricultural Science, 37: 617-621.

15. Chaudhry, A.S. 2000. Rumen degradation in saco in sheep of wheat straw treate with calcium oxid sodium hydroxide and sodium hydroxide plus hydrogen peroxide Animal Feed Science and Technology, 83: 313-323.

16. Danesh Mesgaran, M., M. Malakkhahi, B. Heravi Moussavi, A.R. Vakili and A. Tahmasbi. 2010. In situ ruminal degradation and in vitro gas production of chemically treated sesame stover. Journal of Animal and Veterinary Advances, 9: 2256-2260.

17. Denev, S.A., T.Z. Peeva, P. Radulova, N. Stancheva, G. Staykova, G. Beev, P. Todorova and S. Tchobanova. 2007. Yeast Cultures in Ruminant Nutrition. Bulgarian Journal of Agricultural Science 13: 357-374.

18. Getachew, G., M. Blummel, H. Makkar and K. Becker. 1998. In vitro gas measuring techniques for assessment of nutritional quality of feeds: A review. Animal Feed Science and Technology, 72: 261281.

19. Ghiasvand, M., K. Rezayazdi and M. Dehghan Banadaki. 2011. The effects of different processing methods on chemical composition and ruminal degradability of canola straw and its effect on fattening performance of male Holstein calves. Journal of Animal Science Researches (Agricultural Science). 22(1): 93-104 (In Persian).

20. Goodarzi, N., A.A. Gheisari and M. Toghyani. 2018. The effects of processing and phytase on performance and phosphorus digestibility in broilers. Research on Animal Production, 8(18): 38-46 (In Persian).

21. Haddi, M.L., S. Filacorda, K. Meniai, F. Rollin and P. Susmel. 2003. In vitro fermentation kinetics of some halophyte shrubs sampled at three stage maturity. Animal Feed Science and Technology, 104: $215-225$.

22. Jalc, D., F. Nerud, R. Zitnan and P. Siroka. 1996. The effect of White-rot basidiomycetes on chemical composition and in vitro digestibility of wheat straw. Folka Microbiology, 41: 73-75.

23. Karabulut, A., O. Canbolat, H. Kalkan, F. Gurbuzoll, E. Sucu and I. Filya. 2007. Comparison of in vitro gas production, metabolizable energy, organic matter digestibility and microbial protein production of some legume hays. Asian Australian Journal of Animal Sciences, 20: 517-522.

24. Khorvash, M., S. Kargar, T. Yalchi and G.R. Ghorbani. 2010. Effects of calcium oxide and calcium hydroxide on the chemical composition and in vitro digestibility of soybean straw. Journal of Food, Agriculture and Environment, 8: 356-359. 
25. Kilicalp, N., H. Hizlı and D. Mart. 2017. Chemical composition and rumen degradation characteristics of different Chickpea (Cicer Arietinum L.) lines straw. Turkish Journal of Agriculture-Food Science and Technology, 5(5): 459-463.

26. Lardy, G. and V. Anderson. 2009. Alternative feeds for ruminants, North Dakota State University, Fargo, North Dakota Extention Service, AS-1182, www.ag.ndsu.edu

27. Lena, G. and G.B. Quaglia. 1992. Sacharification and protein enrichment of sugar beet pulp by Pleurotus florida. Biotechnology Technology, 6: 571-574.

28. Liu, J.X., A. Susenbeth and K.H. Sudekum. 2002. In vitro gas production measurements to evaluate interactions between untreated and chemically treated rice straws, grass hay, and mulberry leaves. Journal of Animal Science, 80: 517-524.

29. Makkar, H.P.S., M. Blummel and K. Becker. 1995. Formation of complexes between polyvinyl pyrrolidones or polyethylene glysols and tannins, and their implication in gas production and true digestibility in in vitro techniques. British Journal of Nutrition. 73: 897-913.

30. McDonald, P., R.A. Edwards, J.F.D. Greenhalgh and C.A. Morgan. 1995. Animal nutrition $\left(6^{\text {th }}\right.$ ed.).USA: Longman Scientific and Technical.

31. Mehrabi, A., T. Ghoorchi and S.E. Razavi. 2014. Comparison of chemical composition and rumen degradability among four types of straws treated by Trametes versicolor fungus. Animal Science Journal (Pajouhesh \& Sazandegi), 107: 49-60 (In Persian).

32. Menk, KH. and H. Steingass. 1988. Estimation of the energetic feed value obtained from chemical analysis and in vitro gas production using rumen fluid. Animal Research Development, Separateprint, 28: 7-55.

33. Menke K.H., L. Raab, A. Salewski, H. Steingass, D. Fritz and W. Schneider. 1979. The estimation of the digestibility and metabolizable energy content of ruminant feedstuffs from the gas production when thev are incubated with rumen liauor in vitro. Journal of Agricultural Science. 92: 217-222.

34. National Research Council. 2001. Nutrient requirements of dairy cattle. National Academies Press.

35. Nazem, K., Y. Rouzbehan and S.A. Shojaosadati. 2008. The nutritive value of citrus pulp (lemon andorange) treated with Neurospora sitophila. Journal of Science and Technology of Agriculture and Natural Resources, 12: 495-506.

36. Orskov, E.R. and I. McDonald. 1979. The estimation of protein degradability in the rumen from incubation measurements weighted according to rate of passage. Journal of Agriculture Science, 92: 499-503.

37. Sabzekar, H. 2014. Nutritional value of irradiated millet straw using nylon bag and gas production methods. M.Sc. Thesis of Animal Nutrition. Zabol University, 92 pp (In Persian).

38. Salamatazar, M., R. Salamatdoust-nobar and N. Maheri-sis. 2012. Evaluation of the effects of thymus vulgar on degradability kinetics of canola meal for ruminant using in vitro gas production technique. Journal of Cell and Animal Biology, 6: 164-168.

39. Sarnklong, C., J.W. Cone, W. Pellikaan and W.H. Hendriks. 2010. Utilization of rice straw and different treatments to improve is feed value for ruminants: A review. Asian-Australian Journal of Animal Science, 23: 680-692.

40. SAS. 2003. SAS User's Guide: Statistics, Version 9.1 Edition. SAS Institute, Cary, NC, USA.

41. Shamim, H.M., M.S. Hussain and A. Al-Mahin. 2016. Sold-state fermentation of coconut coir by Pleurotus alocau increases the anti-oxidant properties and autritional value. Biotechnology, 15(6): 141-147.

42. Shojaosadati, S.A., R. Faradouni and A. Madad-Noue. 1999. Protein enrichment of lignocellulosic substrates by solid state fermentation using neurosposastophila Coaservation and Recycling, 27: 7378.

43. Shrivastava, B., S. Thakur, Y.P. Khasa, A. Gupte, A.K. Puniya and R.C. Kuhad. 2011. White-rot fungal conversion of wheat straw to energy rich cattle feed. Biodegradation, 22: 823-831.

44. Theodorou, M.K., B.A. Williams, M.S. Dhanoa, A.B. McAllan and J. France. 1994. A simple gas production method using a pressure transducer to determine the fermentation kinetics of ruminant feeds. Animal Feed Science and Technology, 48: 185-97.

45. Tuyen, V. D., J.W. Cone, J.J.P. Baars, A.S.M. Sonnenberg and W.H. Hendriks. 2012. Fungal strain and incubation period affect chemical composition and nutrient availability of wheat straw for rumen fermentation. Bioresource Technology, 111: 336-342.

46. Van Soest, P.J. 1994. Nutritional ecology of the ruminant. Cornel University Press, Ithaca, New York. $374 \mathrm{pp}$.

47. Yalchi, T. and B. Hajieghrari. 2011. Effect of fungal treatment on chemical composition and in vitro ruminal digestibility of some agricultural residues. African J. Biotechnol, 10(85): 19707-19713.

48. Yang, I., J. Cao, Y. Jin, H.M. Chang, H. Jameel, R. Phillips and Z. Li. 2012. Effect of sodium carbonate pretreatement on chemical compositions and enzymatic saccharification of rice straw. Bioresource Technology, 124: 283-291.

49. Zadrazil, F. 1984. Microbial conversion of lignocellulose into feed. In: Straw and other Fibrous Byproduct as Feed. Sundstol,. F. and Owen, E. eds, 276-292. Elsevier.

50. Zadrazil, F. 1997. Changes in in vitro digestibility of wheat straw during fungal growth and after harvest of oyster mushrooms (Pleurotus spp.) on laboratory and industrial scale. Journal of applied animal Research, 11(1): 37-48. 


\title{
Effect of Chemical and Biological Processing Methods on Chemical Composition, Gas Production Parameters and In Vitro Digestibility of Cicer Arietinum Wastes
}

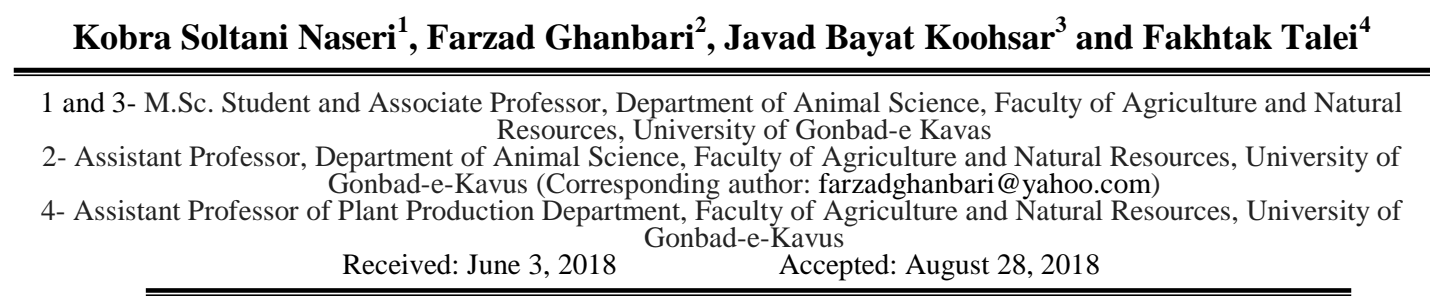

\begin{abstract}
This research was conducted in order to investigate the effect of chemical and biological processing on the chemical composition, gas production parameters and in vitro digestibility of Cicer arietinum wastes in a completely randomized design (7 treatments and 3 replicates). Treatments were: unprocessed Cicer arietinum wastes (control) and processed with water (2.5 liter $/ \mathrm{kg} \mathrm{DM})$, sodium hydroxide $(50 \mathrm{~g} / \mathrm{kg} \mathrm{DM})$, hydrogen peroxide $(114 \mathrm{ml} / \mathrm{kg} \mathrm{DM})$, Aspergillus niger, Trichoderma harzianum and Pleurotus ostreatus fungi $\left(5 \times 10^{5}\right.$ spores $\left./ \mathrm{ml}\right)$. The chemical composition of the samples was determined using the standard methods. Gas production test was used to estimate the parameters of gas production in samples. In vitro digestibility of the samples was determined by the batch culture method. Processing was effective on chemical composition of treatments $(\mathrm{P}<0.03)$. Except for water, the other treatments increased ash and decreased organic matter. Crude protein content was increased by different treatments. The highest amount was observed in Trichoderma harzianum (4.76 percent). Sodium hydroxide decreased neutral detergent fiber compared to control (54 percent versus 56.66 percent). Processing decreased gas production potential and rate. The lowest amount of these traits was observed in Trichoderma harzianum $(162.9 \mathrm{ml} / 200 \mathrm{mg} \mathrm{DM}$ and $0.052 \mathrm{ml} / \mathrm{h}$ respectively). Sodium hydroxide and Aspergillus niger treatments increased $(\mathrm{P}<0.01)$ dry matter and organic matter digestibility $(58$ and 62 percent for dry mater digestibility, 57 and 62 percent for organic matter digestibility respectively). Microbial mass production and its efficiency was increased by the treatments $(\mathrm{P}<0.0003)$. The highest increase was seen in Aspergillus niger treatment (160.28 mg/g DM and 0.48 respectively). Based on the results of this research, sodium hydroxide and Aspergillus niger treatments were effective in improving the nutritional value of Cicer arietinum wastes in laboratory conditions.
\end{abstract}

Keywords: Aspergillus niger, Nutritional value, Cicer arietinum wastes, Trichoderma harzianum, hydrogen peroxide, sodium hydroxide, Pleurotus ostreatus 